\title{
Assessing SB effort via a non-invasive model-based method in mechanically ventilated patients in malaysian ICU hospital
}

\author{
Nor Salwa Damanhuri ${ }^{1}$, Noor Najwa Noor Ariffin ${ }^{2}$, Nor Azlan Othman ${ }^{3}$, Yeong Shiong Chiew ${ }^{4}$, \\ Azrina Md Ralib ${ }^{5}$, Mohd Basri Mat Noor ${ }^{6}$, Paul D. Docherty ${ }^{7}$, J. Geoffrey Chase ${ }^{8}$ \\ ${ }^{1,2,3}$ Faculty of Electrical Engineering, Universiti Teknologi MARA (UiTM), Malaysia \\ ${ }^{4}$ School of Engineering, Monash University, Malaysia \\ ${ }^{5,6}$ Department of Intensive Care, International Islamic University Malaysia Medical Centre, Malaysia \\ ${ }^{7,8}$ Department of Mechanical Engineering, University of Canterbury, New Zealand
}

\section{Article Info \\ Article history: \\ Received Dec 17, 2018 \\ Revised Mar 23, 2019 \\ Accepted Apr 14, 2019}

\section{Keywords:}

ARDS

Lung mechanics

Mathematical modelling

Mechanical ventilation

Spontaneous breathing

\begin{abstract}
Patients with Acute Respiratory Distress Syndrome (ARDS) required mechanical ventilation (MV) for breathing support. However, some MV patients encountered spontaneous breathing (SB) efforts while fully sedated which can obscure the true underlying respiratory mechanics of these patients. Thus, a model-based method is required to reconstruct the missing pressure and calculate the breathing effort that produced by the patients without additional clinical protocols or invasive procedure. In this paper, results of spontaneous breathing effort in Malaysian critically-ill patients adopting the developed pressure reconstruction model are presented. By using the pressure reconstruction model, the SB affected pressure waveform is reconstructed to approximate true respiratory mechanics and quantifies the SB effort. The SB breathing efforts were computed and compared with the results from Christchurch Hospital, New Zealand. The substitute measure of SB effort can be indicated from the difference between the reconstructed and unreconstructed pressure. Results shows that all patients from both cohorts exhibited SB effort with the highest SB effort at $11.48 \%$ for Malaysian patient and $21.07 \%$ for Christchurch patient. Overall, the well-developed non-invasive pressure reconstruction method is able to measure the SB effort produced by Malaysian MV patients that help the clinicians in selecting the optimal MV setting. This first non-invasive guidance in selecting the optimal setting of MV in Malaysia is potentially reduced the ICU cost and improve the MV management in Malaysian hospital.
\end{abstract}

Copyright @ 2019 Institute of Advanced Engineering and Science. All rights reserved.

\section{Corresponding Author:}

Nor Salwa Damanhuri,

Faculty of Electrical Engineering,

Universiti Teknologi MARA (UiTM),

Pulau Pinang, Permatang Pauh 13500,

Pulau Pinang, Malaysia.

Email: norsalwa071@ppinang.uitm.edu.my

\section{INTRODUCTION}

Acute respiratory Distress Syndrome patients require mechanical ventilation (MV) as a breathing support $[1,2]$. However, MV can further injured the damaged lungs known as a ventilator-induced lung injury (VILI) if it is not properly managed [3, 4]. The main goal of MV in ARDS patient is to minimize any additional damage while preserving sufficient gas exchange [5].

Characterization of patient-specific state and reaction to treatment can be evaluated from the respiratory mechanics $[6,7]$. Respiratory mechanics refers to the declaration of lung function through 
measures of flow and pressure. However, during ventilator supported breaths, the alteration of airway pressure waveforms can occur in many patients that exhibited spontaneous breathing efforts. Consequently, the model-based identification will be obscured from showing the true, original respiratory mechanics required for a better MV guidance.

Reverse-triggering of patient breathing efforts, induced by ventilator, is referred to as an asynchrony where SB exhibits during a ventilator supported breathing cycle that inaccurately measured the true underlying of the respiratory mechanics $[8,9]$. As shown in Figure 1, the reduction in the patient's airway pressure waveform were formed by the patient's own breathing effort. Thus, this lead to a poor model fitting when the existing developed model were applied to calculate the lung elastance.

Particularly, the decrement in airway pressure of a specific volume would also decrease the value of respiratory elastance due to the SB exhibited by the patient. This is because the patient inhalation effort produces negative elastance component $[6,10]$. In consequence, the acknowledged parameters are not the true representation of the real underlying mechanics since the input of variable inhalation specifically produced by the patient was not taken into account. On top of that, patients with SB effort may not be ventilated correctly based on their ability to breathe spontaneously. They may need different type of ventilator mode that provides mechanical breaths to a patient such as Synchronized Intermittend Mandatory Ventilation (SIMV) [11].

Previously, breathing effort was estimated invasively by inserting the balloon catheter [12, 13]. Thus, SB patients required a non-invasive pressure reconstruction model that is able to reconstruct the missing pressure and calculate the SB effort for a better MV management. Damanhuri et. al has developed a pressure reconstruction model that is able to assses the breathing effort in SB ventilated patients without additional tools and protocol by using the Christchurch Hospital data [14]. Hence, this study aims to assess, evaluate and compare the SB ventilated patients in Malaysian ICU setting, International Islamic University (IIUM) Hospital against Christchurch Hospital, New Zealand. This would be the first non-invasive model development for Malaysian ICU patients that is beneficial in managing the MV setting specifically for SB patients which could guide clinicians in better MV management and reduce the ICU cost at the same time.
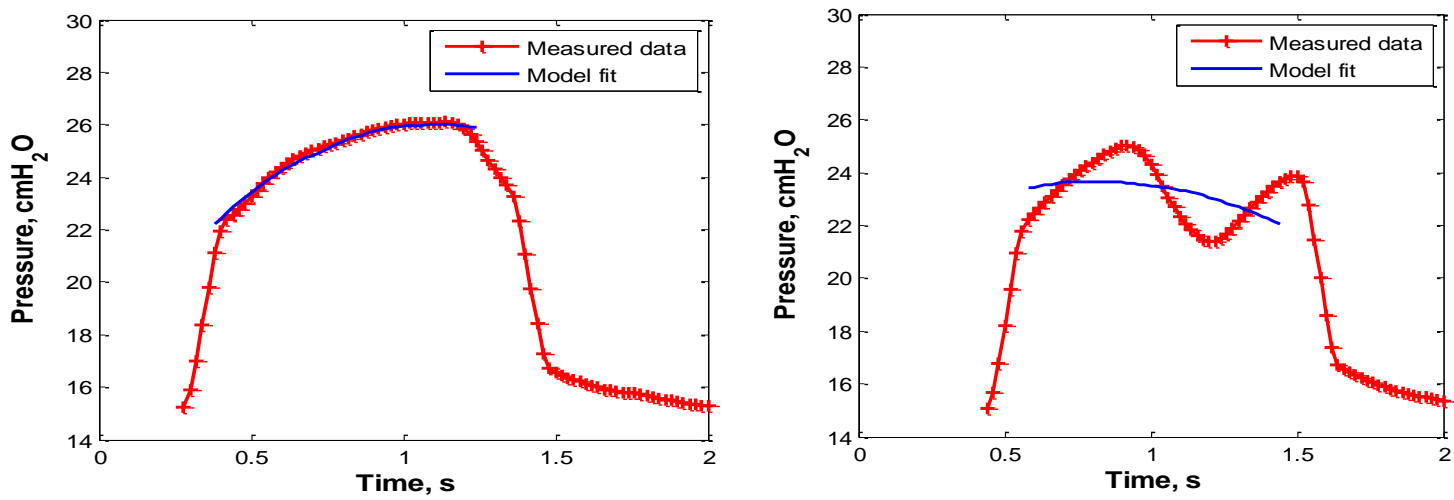

Figure 1. Differentiation of (left) a normal airway pressure waveform with good model fitting to (right) an airway pressure waveform with breathing effort that leads to a poor model fit to the pressure waveform [14]

\section{RESEARCH METHOD}

\subsection{Patient Data and Analysis}

The data used in this study was obtained from 883 breathes aggregated from 7 mechanically ventilated patients admitted to the intensive care unit (ICU) between August 2017 and October 2017 at the IIUM Medical Centre, Malaysia with respiratory failure [15]. There were also 635 breathes from 5 MV patients of Christchurch Hospital, New Zealand ICU between April 2014 and November 2014 [16, 17]. The patients involved were ventilated using Puritan Bennett PB980 ventilator (Covidien, Boulder, CO, USA). Data collection was performed by using a CURE soft system [18] where airway pressure and flow were recorded for each patient as shown in Figure 2. The inclusion criteria of the patients enrolled were those aged above 16, requiring invasive $\mathrm{MV}$ and with ratio of oxygen partial pressure to fraction of inspired oxygen $\left(\mathrm{P}_{\mathrm{a}} \mathrm{O} 2 / \mathrm{F}_{\mathrm{i}} \mathrm{O} 2\right)$ less than $300 \mathrm{mmHg}$. While the elimination conditions comprise of patients who mostly in 24 hours, are to be withdrawn from MV, patients with a great injury of spinal cord with motor function loss, and also patients who are not expected to last for more than 72 hours or in a dying state. The trial number for IIUM patients is IREC66 while for the Christchurch Hospital patients is 
ACTRN12613001006730. Table 1 and 2 shows the demographics of the patients enrolled for both cohorts in this study.

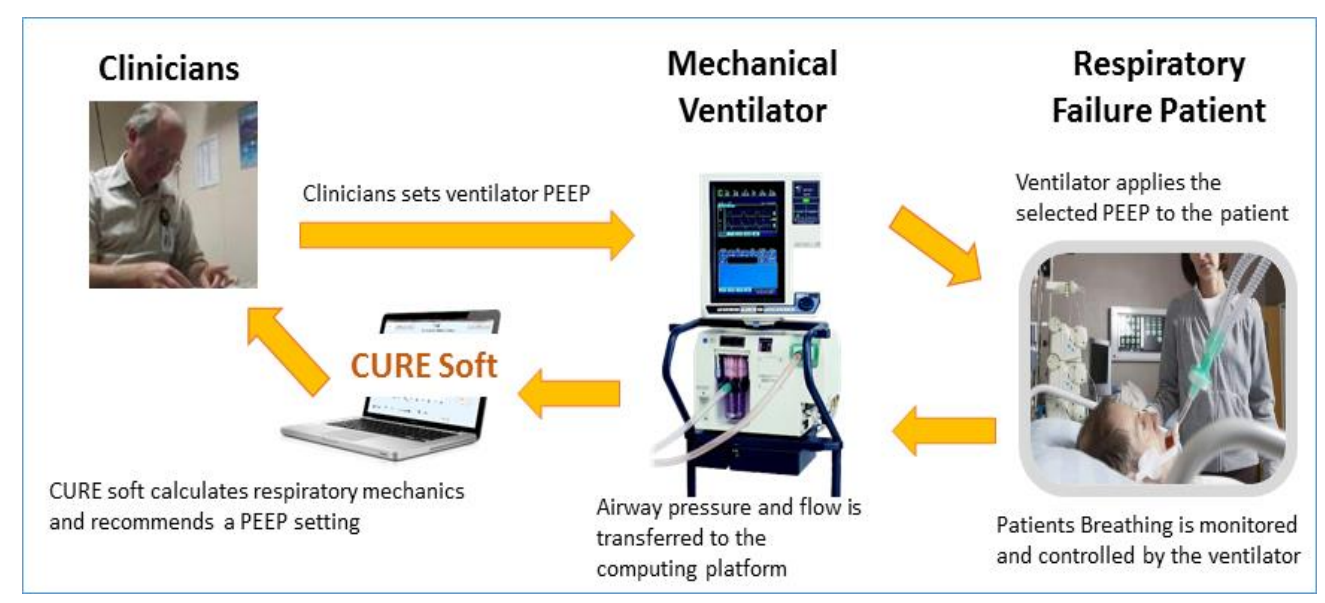

Figure 2. CURE software application for respiratory mechanics monitoring in the ICU [18]

Table 1. Characteristics of Patients in IIUM Hospital, Malaysia

\begin{tabular}{cccc}
\hline Patient no. & Gender & Age & Clinical Diagnostic \\
\hline M1 & Female & 43 & Hospital Acquired Pneumonia (HAP) \\
M2 & Male & 54 & Hospital Acquired Pneumonia (HAP) \\
M3 & Male & 52 & Lung Cancer \\
M4 & Male & 64 & Hospital Acquired Pneumonia (HAP) \\
M5 & Female & 63 & Hospital Acquired Pneumonia (HAP) \\
M6 & Female & 73 & Septic Shock \\
M7 & Female & 64 & Community Acquired Pneumonia \\
\hline
\end{tabular}

Table 2. Characteristics of patients in Christchurch Hospital, New Zealand

\begin{tabular}{cccc}
\hline Patient No. & Gender & Age & Clinical Diagnostic \\
\hline C1 & Female & 53 & Faecal peritonitis \\
C2 & Male & 71 & Hospital Acquired Pneumonia (HAP) \\
C3 & Male & 60 & Pneumonia \\
C4 & Male & 36 & Pneumonia \\
C5 & Male & 61 & Pneumonia \\
\hline
\end{tabular}

\subsection{Time Varying Elastance Model}

Respiratory mechanics can be used to represent patient-specific condition and feedback to treatment, and are conservatively estimated using a single-compartment linear lung model $[11,19]$ as in (1):

$$
P_{a w}(t)=R_{r s} Q(t)+E_{r s}(t) V(t)+P_{0}
$$

The airway pressure is labelled as $\mathrm{P}_{\mathrm{aw}}$, the respiratory system elastance is defined as $\mathrm{E}_{\mathrm{rs}}$, while $\mathrm{V}$ is the lung volume, the respiratory system resistance is denoted as $\mathrm{R}_{\mathrm{rs}}$. $\mathrm{Q}$ means the airway flow, and when the intrinsic positive end expiratory pressure (PEEP) is none, the offset pressure or PEEP is $\mathrm{P}_{\mathrm{o}}$. By applying an integral based-method, the values for $E_{r s}$ and $R_{r s}$ can be easily calculated with flow data and inspiratory $P_{a w}$ $[20,21]$.

$$
\int P_{a w}(t) d t=R_{r s} \int Q(t) d t+E_{r s}(t) \int V(t) d t+\int P_{0} d t
$$

Next, for every PEEP data level, with reference to the value of $\mathrm{R}_{\mathrm{rs}}$, the lung resistance average value $\left(R_{a v e}\right)$ is calculated. While the identification of the time-varying lung elastance, $E_{\text {drs }}$ can be made using [22]:

$$
E_{d r s}(t)=\frac{P_{a w}(t)-P_{0}-\left(R_{a v e} Q(t)\right)}{V(t)}
$$


The area under the curve of $E_{d r s}\left(A U C E_{d r s}\right.$ ) is then identified for every breathing cycle as a substitute of respiratory elastance over the breath.

\subsection{Pressure Reconstruction Model}

Pressure recontruction model was first developed by Damanhuri et. al [14]. Due the SB effort produced by the MV patients even if sedated, it thus creates a significantly lower identified lung elastance, which inaccurately measure the respiratory mechanics of MV patients. Thus, with pressure reconstruction model, it helps to reconstruct the missing pressure and able to estimate the true underlying respiratory mechanics of SB patients as shown in Figure 3 [14, 23].

The airway pressure's gradient during exhalation cycle can be the determined by referring to points $a$ and $b$ as seen in Figure 3b. The connecting line of point $a$ and $b$ is extrapolated further to the point $c_{l}$ where it reaches the same value as the highest pressure which is defined as $c_{2}$ now. The peak and end inspiratory gradient are connected through a line from point $c_{1}$ and $c_{2}$.

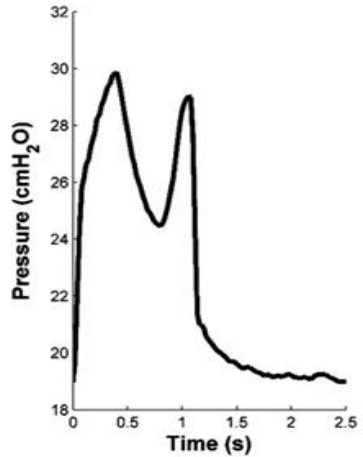

(a)

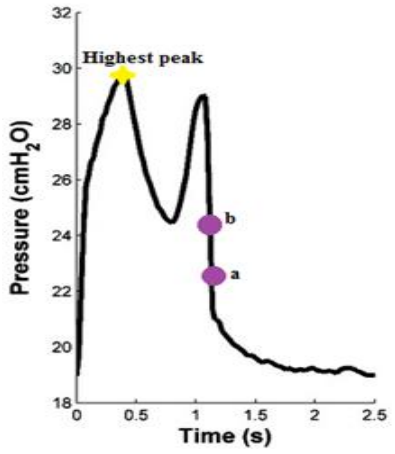

(b)

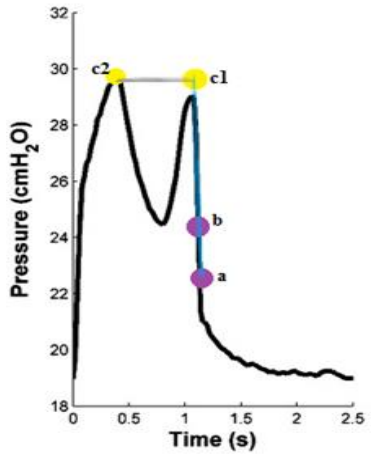

(c)

Figure 3. The steps on the reconstruction process (a) The airway pressure in SB patient.

(b) The point $a, b$ and the highest peak are identified. (c) The slope of point $a$ and point $b$ is extrapolated until point $c_{1}$, which has the same pressure value as the highest peak denoted as $c_{2}$.

\subsection{Estimation of Spontaneous Breathing Effort}

When a mechanically ventilated patient exhibits SB effort during MV, the contraction of diaphragm generates a negative pressure in the pleural space, which generates a drop in the airway pressure. The level of SB effort can be assessed after the pressure has been reconstructed by calculating the difference of AUC $E_{\mathrm{drs}}$ between the reconstructed and unreconstructed pressure, which is denoted as the substitute of SB effort.

As shown in Figure 4, $\mathrm{A}_{1}$ is defined as the area of the entrained pressure waveform which has lower overall time varying elastance (AUC $E_{\text {drs }}$ ) compared to the reconstructed waveform $[14,23]$. The value of elastance could be led higher with an addition of $\mathrm{A}_{2}$ which is defined as the area of missing pressure. This would reduced the effect of SB effort thus, the identification of SB effort in percentage can be made as [14]:

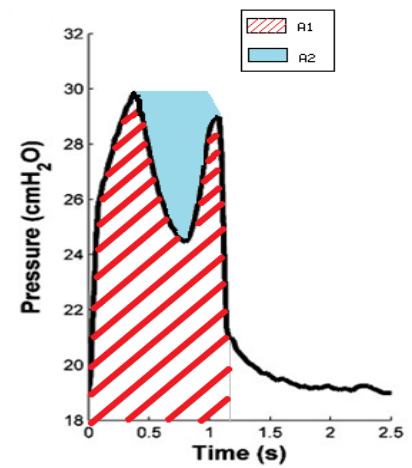

Figure 4.The shaded areas of the entrained pressure waveform, $\mathrm{A}_{1}$ and the missing pressure waveform, $\mathrm{A}_{2}$. 
SB effort $=\frac{A_{2}}{A_{1}+A_{2}} \times 100 \%$

\section{RESULTS AND ANALYSIS}

All MV patients who are usually in full volume controlled mode and fully sedated may produce SB efforts that affect the patients' respiratory mechanics as they vary the normal airway pressures [14, 24]. Figure 5 shows the unreconstructed airway pressure waveforms for Patient $\mathrm{C} 1$ and Patient M1 at PEEP of 15 and $4 \mathrm{cmH}_{2} \mathrm{O}$ respectively. It can bee seen that both Patient $\mathrm{M} 1$ and $\mathrm{C} 1$ encountered some pressure reductions or an entrainent in the airway pressure waveform that occurred due to the SB actions.

Thus, pressure reconstruction method is applied and the results are depicted in Figure 6. As shown in Figure 6, the pressure reconstruction method is able to reconstruct the missing pressure and produce an almost perfect airway pressure in Patient C1. In contrast, for Patient M1, the pressure reconstruction method is not perfectly reconstructed the missing pressure. This might be due to the different ventilation mode applied [14, 24].

Furthermore, with the pressure reconstruction method, the SB effort for all patients from both cohorts were able to be estimated as tabulated in Table 3 and 4 respectively. These results show the ability of this reconstruction method to estimate the level of SB effort in MV patients non-invasively and does not require any additional clinical protocols. From Table 3, it shows that Patient M1 exhibited the highest level of SB effort as compared to other patients from the same cohort which is $11.48 \%$ at $\mathrm{PEEP} 4 \mathrm{cmH}_{2} \mathrm{O}$. On the other hand, Patient $\mathrm{C} 1$ from Christchurch Hospital exhibited the highest level of SB of $21.07 \%$ at PEEP 4 $\mathrm{cmH}_{2} \mathrm{O}$. Patients in IIUM were ventilated at lower PEEP level and thus, not much breathing effort can be seen as compare to the patients from Christchurch Hospital. Thus, from this research, it can be perceived that, regardless of being anesthetized and ventilated in full controlled mode, the SB affected breaths for all patients can still be measured and estimated as tabulated in Tables 3 and 4.
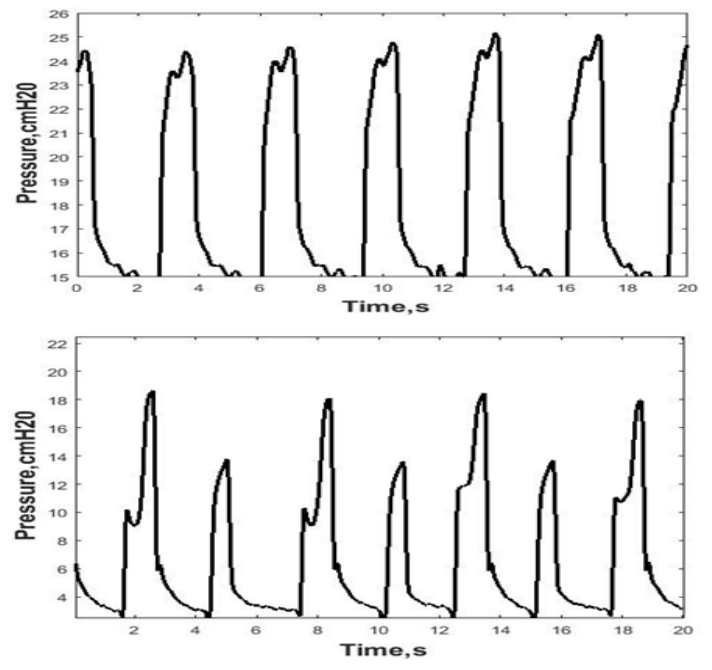

Figure 5. The unreconstructed airway pressure waveforms. Top: Patient $\mathrm{C} 1$ at PEEP of $15 \mathrm{cmH}_{2} \mathrm{O}$. Bottom: Patient $\mathrm{M} 1$ at PEEP of $4 \mathrm{cmH}_{2} \mathrm{O}$
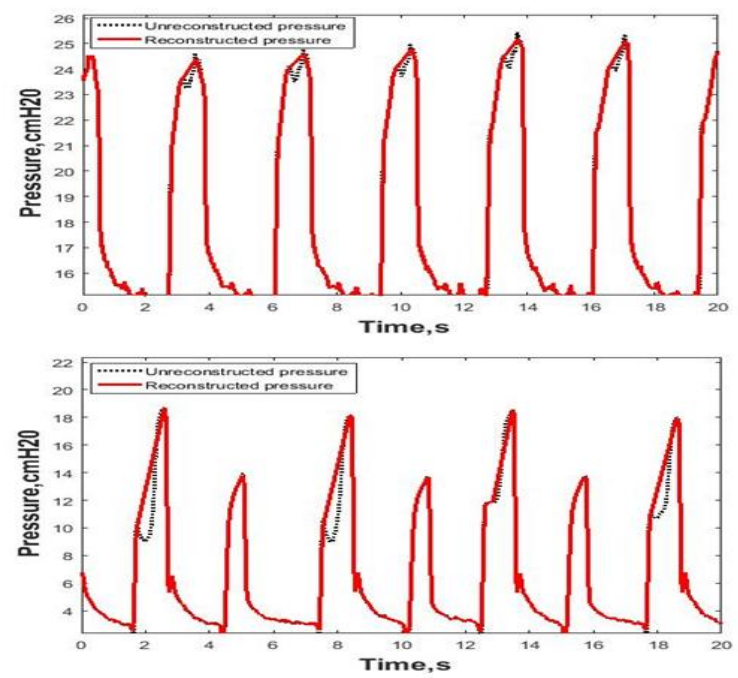

Figure 6. The reconstructed airway pressure waveforms. Top: Patient $\mathrm{C} 1$ at PEEP of $15 \mathrm{cmH}_{2} \mathrm{O}$. Bottom: Patient M1 at PEEP of $4 \mathrm{cmH}_{2} \mathrm{O}$.

On the other hand, the analyzation of the variability of $E_{\mathrm{drs}}$ through all PEEP in the 7 patients from IIUM Hospital and 5 patients from Christchurch Hospital were done as this project also aims to compute the variability in the respiratory mechanics. Due to the SB effort, it produced a negative elastance in the patient's lung [25]. Thus, this will lead to the variability of the lung elastance in MV patients. The study of the elastance's variability in the patients was done by applying [1] and for each cycle of breath, the AUC E $\mathrm{drs}_{\mathrm{ds}}$ was assessed and analysed. As seen in Figure 7, for each patient, the AUC Edrs differs at each PEEP level. Precisely, Christchurch patients at $\mathrm{PEEP}=15 \mathrm{cmH}_{2} \mathrm{O}$ produced lower variability of elastance with median $=$ 4 and IQR value of [3.29 - 4.58] compared to Malaysian patients that has the highest range of elastance at $\mathrm{PEEP}=3 \mathrm{cmH}_{2} \mathrm{O}$ with median of 29.6 and IQR value of [23.19-34.38]. From the results of the median and 
IQR through all levels of PEEP, it shows that Christchurch patients have higher range of variability of AUC $\mathrm{E}_{\mathrm{drs}}$. These results were already predicted as Christchurch patients specifically patient $\mathrm{C} 1$ exhibited more SB effort compared to Malaysian patient as shown in previous results above. This concludes that each patient exhibited different levels of SB effort independent from MV setting, indicating the need for non-invasive, real time assessment of SB effort.

Table 3. Characteristics of Patients in IIUM Hospital, Malaysia

\begin{tabular}{ccccc}
\hline $\begin{array}{c}\text { Patient } \\
\text { no. }\end{array}$ & $\begin{array}{c}\text { PEEP } \\
\left(\mathrm{cmH}_{2} 0\right)\end{array}$ & $\begin{array}{c}\text { Breathing } \\
\text { Cycle }\end{array}$ & $\begin{array}{c}\text { SB Affected } \\
\text { Cycle }\end{array}$ & $\begin{array}{c}\text { SB Effort } \\
(\%)\end{array}$ \\
\hline \multirow{2}{*}{ M1 } & 4 & 63 & 37 & 11.48 \\
& 3 & 74 & 40 & 9.91 \\
M2 & 9 & 168 & 12 & 1.02 \\
M3 & 10 & 14 & 4 & 4.23 \\
M4 & 16 & 90 & 24 & 0.02 \\
M5 & 9 & 65 & 44 & 7.19 \\
& 9 & 68 & 42 & 7.47 \\
M6 & 8 & 155 & 30 & 1.02 \\
& 9 & 10 & 4 & 0.09 \\
M7 & 10 & 90 & 48 & 0.21 \\
& 11 & 28 & 16 & 4.59 \\
& & 58 & 37 & 7.77 \\
\hline
\end{tabular}

Table 4. Characteristics of Patients in Christchurch Hospital, New Zealand

\begin{tabular}{ccccc}
\hline $\begin{array}{c}\text { Patient } \\
\text { no. }\end{array}$ & $\begin{array}{c}\text { PEEP } \\
\left(\mathrm{cmH}_{2} \mathrm{O}\right)\end{array}$ & $\begin{array}{c}\text { Breathing } \\
\text { Cycle }\end{array}$ & $\begin{array}{c}\text { SB } \\
\text { Affected } \\
\text { Cycle }\end{array}$ & $\begin{array}{c}\text { SB Effort } \\
(\%)\end{array}$ \\
\hline C1 & 15 & 106 & 106 & 21.07 \\
C2 & 16 & 127 & 93 & 12.67 \\
C3 & 13 & 112 & 44 & 0.12 \\
C4 & 14 & 18 & 11 & 0.15 \\
C5 & 22 & 130 & 113 & 0.76 \\
& 13 & 34 & 6 & 0.05 \\
\end{tabular}
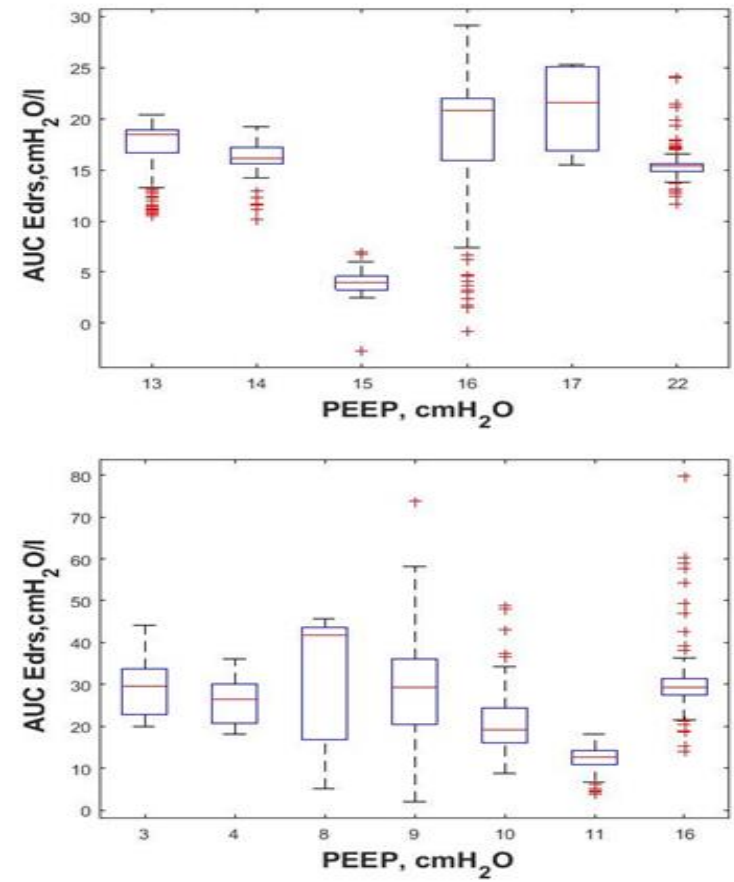

Figure 7. The distribution of AUC $E_{\text {drs }}$ at every PEEP level. Top: Data from all 5 patients in Christchurch Hospital. Bottom: Data from all 7 patients in IIUM Medical Centre 


\section{CONCLUSION}

Patients on ventilator support who are not fully sedated can exhibit SB effort which may cause an alteration in the pressure waveforms recorded during the MV. This will result in an inaccurate estimation of true respiratory mechanics that will eventually lead to further lung damage. Thus, a computationally simple, non-invasive modelling of pressure reconstruction method is proposed based on data IIUM Hospital and compared with the Christchurch Hospital. The results show that the proposed model was able to estimate the SB effort produced by the MV patients although they were fully sedated. Results shows that all patients from both cohorts exhibited SB effort with the highest SB effort at $11.48 \%$ for Malaysian patient. Thus, this method allows more accurate estimation of mechanical properties that can be used by clinicians in guiding patient-specific MV care as it takes into account the existence of SB that masked the true concealed respiratory mechanics. It also opens a new venture in monitoring the lung elastance performance especially for SB patients in Malaysian Hospital.

\section{ACKNOWLEDGEMENTS}

Ministry of Education, Malaysia, Universiti Teknologi MARA UiTM and the New Zealand Health Research Council, Project PulMoDS HRC reference 13/213 for providing research funding. EU FP7/RSNZ Marie Curie IRSES for further funding.

\section{REFERENCES}

[1] Ranieri VM, Rubenfeld GD, Thompson BT, Ferguson ND, Caldwell E, Fan E, et al. Acute respiratory distress syndrome: the Berlin Definition. JAMA. 2012;307(23):2526-33. Epub 2012/07/17.

[2] Sweeney RM, McAuley DF. Acute respiratory distress syndrome. Lancet. 2016;388(10058):2416-30. Epub 2016/05/03.

[3] Slutsky AS. Lung Injury Caused by Mechanical Ventilation*. Chest. 1999;116(suppl 1):9S-15S.

[4] Slutsky AS, Ranieri VM. Ventilator-induced lung injury. N Engl J Med. 2013;369(22):2126-36. Epub 2013/11/29.

[5] Sundaresan A, Yuta T, Hann CE, Geoffrey Chase J, Shaw GM. A minimal model of lung mechanics and modelbased markers for optimizing ventilator treatment in ARDS patients. Computer Methods and Programs in Biomedicine. 2009;95(2):166-80.

[6] Lucangelo U, Bernabe F, Blanch L. Lung mechanics at the bedside: make it simple. Current Opinion in Critical Care. 2007;13(1):64-72.

[7] Bellani G, Laffey JG, Pham T, Fan E, Brochard L, Esteban A, et al. Epidemiology, Patterns of Care, and Mortality for Patients With Acute Respiratory Distress Syndrome in Intensive Care Units in 50 Countries. JAMA. 2016;315(8):788-800. Epub 2016/02/24.

[8] Blanch L, Villagra A, Sales B, Montanya J, Lucangelo U, Lujan M, et al. Asynchronies during mechanical ventilation are associated with mortality. Intensive Care Med. 2015;41(4):633-41. Epub 2015/02/20.

[9] Akoumianaki E, Lyazidi A, Rey N, Matamis D, Perez-Martinez N, Giraud R, et al. Mechanical Ventilation-Induced Reverse-Triggered BreathsReverse TriggeringA Frequently Unrecognized Form of Neuromechanical Coupling. CHEST Journal. 2013;143(4):927-38.

[10] Bates JHT. Lung Mechanics: An Inverse Modeling Approach: Cambridge University Press; 20092009.

[11] Chiew YS, Pretty C, Docherty PD, Lambermont B, Shaw GM, Desaive T, et al. Time-Varying Respiratory System Elastance: A Physiological Model for Patients Who Are Spontaneously Breathing. PloS one. 2015;10(1).

[12] Talmor D, Sarge T, Malhotra A, O'Donnell CR, Ritz R, Lisbon A, et al. Mechanical Ventilation Guided by Esophageal Pressure in Acute Lung Injury. New England Journal of Medicine. 2008;359(20):2095-104.

[13] Khirani S, Polese G, Aliverti A, Appendini L, Nucci G, Pedotti A, et al. On-line monitoring of lung mechanics during spontaneous breathing: a physiological study. Respiratory medicine. 2010;104(3):463-71.

[14] Damanhuri NS, Chiew YS, Othman NA, Docherty PD, Pretty CG, Shaw GM, et al. Assessing respiratory mechanics using pressure reconstruction method in mechanically ventilated spontaneous breathing patient. Comput Methods Programs Biomed. 2016;130:175-85. Epub 2016/05/22.

[15] Chiew YS, Chase JG, Arunachalam G, Tan CP, Loo NL, Chiew YW, et al. Clinical Application of Respiratory Elastance (CARE Trial) for Mechanically Ventilated Respiratory Failure Patients: A Model-based Study. IFACPapersOnLine. 2018;51(27):209-14.

[16] Davidson SM, Redmond DP, Laing H, White R, Radzi F, Chiew YS, et al., editors. Clinical Utilisation of Respiratory Elastance (CURE): Pilot Trials for the Optimisation of Mechanical Ventilation Settings for the Critically Ill. World Congress; 2014.

[17] Major V, Simon C, Redmond D, Beatson A, Glassenbury D, Chiew YS, et al. Assessing respiratory mechanics of reverse-triggered breathing cycles-case study of two mechanically ventilated patients. IFAC-PapersOnLine. 2015;48(20):505-10.

[18] Szlavecz A, Chiew YS, Redmond D, Beatson A, Glassenbury D, Corbett S, et al. The Clinical Utilisation of Respiratory Elastance Software (CURE Soft): a bedside software for real-time respiratory mechanics monitoring and mechanical ventilation management. BioMedical Engineering OnLine. 2014;13(1):140. 
[19] Lauzon A, Bates J. Estimation of time-varying respiratory mechanical parameters by recursive least squares. J Appl Physiol. 1991;71(3):1159-65.

[20] Docherty PD, Chase JG, David T. Characterisation of the iterative integral parameter identification method. Medical \& biological engineering \& computing. 2012;50(2):127-34.

[21] Hann CE, Chase JG, Lin J, Lotz T, Doran CV, Shaw GM. Integral-based parameter identification for long-term dynamic verification of a glucose-insulin system model. Computer Methods and Programs in Biomedicine. 2005;77(3):259-70.

[22] Chiew YS, Poole SF, Redmond DP, van Drunen EJ, Damanhuri NS, Pretty C, et al., editors. Time-Varying Respiratory Elastance for Spontaneously Breathing Patients. 19th World Congress of the International Federation of Automatic Control; 2014 August 24-29; Cape Town, South Africa.

[23] Chiew YS, Tan CP, Chase JG, Chiew YW, Desaive T, Ralib AM, et al. Assessing mechanical ventilation asynchrony through iterative airway pressure reconstruction. Computer Methods and Programs in Biomedicine. 2018;157:217-24.

[24] Kannangara DO, Newberry F, Howe S, Major V, Redmond D, Szlavecs A, et al. Estimating the true respiratory mechanics during asynchronous pressure controlled ventilation. Biomedical Signal Processing and Control. 2016;30:70-8.

[25] Damanhuri NS, Chiew YS, Docherty PD, Othman NA, Shaw GM, Desaive T, et al. Negative Lung Elastance in Mechanically Ventilated Spontaneously Breathing Patient. IFAC-PapersOnLine. 2017;50(1):15179-84.

\section{BIOGRAPHIES OF AUTHORS}

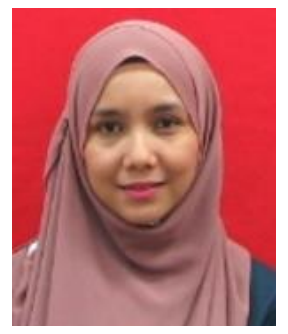

Nor Salwa Damanhuri received her B.Sc. (Hons) in Electrical \& Electronics Engineering from Universiti Tenaga Nasional (UNITEN) Malaysia. In 2004, she received the Excellence Scheme Programme from MARA to pursue her MSc. in Control Systems Engineering in University of Sheffield, United Kingdom. She had 4 years experiences as a Product Engineer in Freescale Semiconductor Malaysia (fka Motorola Semiconductor) before embark her journey as a lecturer at the Universiti Teknologi MARA (UiTM) Pulau Pinang. She obtained her PhD in Bioengineering from University of Canterbury, New Zealand in 2015. Currently, she is a senior lecturer in Faculty of Electrical Engineering, UiTM Pulau Pinang. Her research interests include lung mechanics, system identification methods, modelling for type 2 diabetic patients and solar PV system.

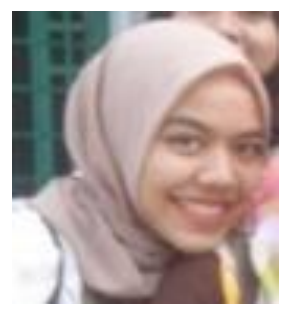

Noor Najwa Noor Ariffin received her Bachelor degree in Electrical \& Electronics Engineering from Universiti Teknologi MARA (UiTM) Pulau Pinang. At present, she is an engineer in one of the multinational company in Penang.

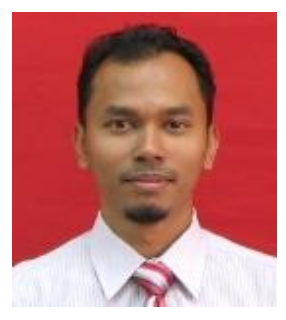

Nor Azlan Othman received her B.Sc. (Hons) in Electrical \& Electronics Engineering from Universiti Tenaga Nasional (UNITEN) Malaysia. In 2004, he received the Excellence Scheme Programme from MARA to pursue his MSc. in Control Systems Engineering in University of Sheffield, United Kingdom. He had several years of experiences as a R\&D Engineer in Sony and Motorola Malaysia. He obtained his $\mathrm{PhD}$ in Bioengineering from University of Canterbury, New Zealand in 2015. Currently, he is a senior lecturer in Faculty of Electrical Engineering, UiTM Pulau Pinang. His research interests include physiological modelling, parameter identification, insulin sensitivity for type 2 diabetes, renewable energy and control systems.

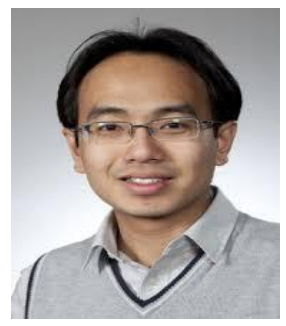

Yeong Shiong Chiew received his BEng and MEng from Universiti Teknologi Malaysia (UTM) in 2007 and 2010, respectively. He then obtained his PhD at the University of Canterbury (UC), New Zealand, investigating into model-based treatment for critically ill patients. He was a postdoctoral fellow at Centre for Bioengineering at UC from 2013 to 2015, researching into a range of biomedical engineering applications. He is currently a senior lecturer in Monash University Malaysia, working on model-based clinical research and clinical trials in regional hospitals. His work spans from the development of physiological models, system design, and system identification methods, to the creation of decision metrics used in clinical environment. 


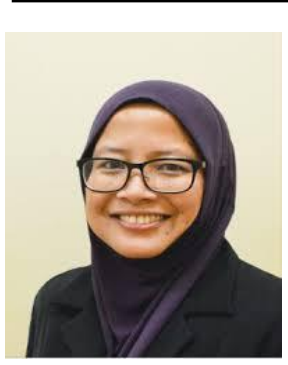

Azrina Md Ralib obtained her Bachelor of Medical Science degree from the University of St. Andrews, UK and Bachelor of Medicine and Bachelor of Surgery (MBChB) degree from the University of Manchester, UK. She later joined the Department of Anaesthesiology and Intensive Care in 2002 as trainee lecturer, and started her anaesthesiology training at the Universiti Sains Malaysia, Kubang Kerian. She graduated as Clinical Specialist in Anaesthesiology in 2006 with several awards to her name, including the Esmeron ${ }^{\circledR}$ M.Med. Anaesthesiology USM Award for best student performance in Master of Medicine (Anaesthesiology), and Research Fellowship Award for original and significant research by The Heart Foundation of Malaysia. She then further her study at doctoral level at the University of Otago, New Zealand where she was awarded PhD in 2013. Presently, she is an Associate Professor at the Department of Anaesthesiology of IIUM. She is also a full time practicing Consultant Anaesthesiologist at the IIUM Medical Centre, Kuantan.

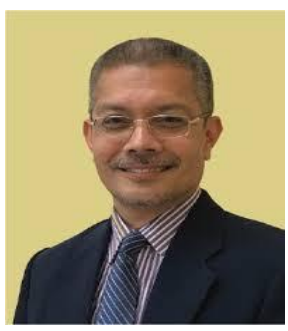

Mohd Basri Mat Nor is a Senior Lecturer at Department of Anaesthesiology and Intensive Care, Kulliyyah (School) of Medicine, International Islamic University Malaysia (IIUM). He is also a Senior Consultant in Intensive Care and Head of Intensive Care Service at IIUM Medical Centre. $\mathrm{He}$ is one of the pioneers in intensive care medicine specialist in the country and has been elected as President of Malaysian Society of Intensive Care since 2017. He obtained his medical degree from Royal College of Surgeons in Ireland (RCSI) and National University of Ireland, Dublin in 1990. He obtained M.Med (Anaesthesiology) from University of Malaya in 1998 and then pursued subspecialty training (fellowship) in intensive care medicine for four years in Australia. After serving Hospital Kuala Lumpur for two years as specialist, he joined IIUM in 2000. He is one of the members of writing committee for national ICU protocol and expert panel for national ICU antimicrobial guidelines. Currently he is the Head of Research and Innovation (HORI) of IIUM Medical Faculty. His research areas include sepsis biomarkers, nutrition in critically ill, mechanical ventilation in acute respiratory failure and Antibiotics PK/PD.

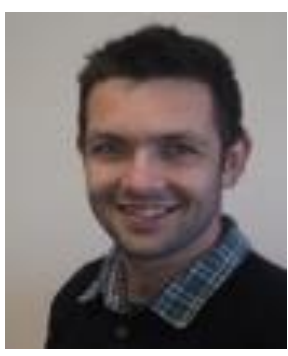

Paul David Docherty holds a PhD in Mechanical Engineering concentrating on numerical methods in physiology, a BE (hons) mechanical and is qualified floor and wall tiler. Paul Docherty recieved a BE (hons) in 2007 and a $\mathrm{PhD}$ in 2011 from the University of Canterbury (New Zealand). He earned a trade qualification in 2003. He is currently an associate professor at the University of Canterbury, New Zealand and has research interests in parameter identification and bioegnineering.

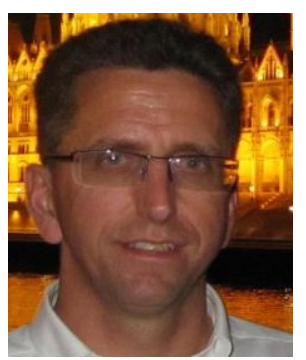

James Geoffrey Chase received his Bachelor in science from Case Western Reserve University, Cleveland, Ohio in 1986 and his Masters in Science and PhD from Stanford University in 1991 and 1996. He spent 6 years working for General Motors and a further 5 years consulting in Silicon Valley, including positions at Xerox PARC, GN ReSound, Hughes Space and Communications and Infineon Technologies AG, before the University of Canterbury. Currently, he holds a position of Distinguished Professor in University of Canterbury, New Zealand. His research interests include automatic control, physiological systems dynamics, structural dynamics and vibrations, dynamic and systems modeling. 